\title{
miR-409-3p sensitizes colon cancer cells to oxaliplatin by inhibiting Beclin-1-mediated autophagy
}

\author{
SHIFAN TAN ${ }^{1 *}$, HUIJUAN SHI ${ }^{2 *}$, MINGCHEN BA $^{1}$, SHENGQV LIN $^{1}$, \\ HONGSHENG TANG ${ }^{1}$, XIAOQI ZENG ${ }^{1}$ and XIANGLIANG ZHANG ${ }^{1}$ \\ ${ }^{1}$ Department of Abdominal Surgery (Section 2), Affiliated Cancer Hospital of Guangzhou Medical University, \\ Guangzhou, Guangdong 510095; ${ }^{2}$ Department of Pathology, The First Affiliated Hospital \\ of Sun Yat-Sen University, Guangzhou, Guangdong 510080, P.R. China
}

Received April 4, 2015; Accepted January 19, 2016

DOI: $10.3892 / \mathrm{ijmm} .2016 .2492$

\begin{abstract}
The chemoresistance of colon cancer cells limits the efficacy of chemotherapy. miR-409-3p has been shown to be downregulated in various types of cancer. In the present study, we examined the role of miR-409-3p in colon cancer as well as the effects of miR-409-3p on the sensitivity of colon cancer cells to oxaliplatin. The expression of miR-409 was significantly downregulated in the human colon cancer cell lines compared with the normal colon epithelial cells. Importantly, the miR-409-3p expression levels were lower in human colon cancer patient samples than in normal colon tissues. Moreover, we observed a negative correlation between the miR-409-3p levels and resistance to oxaliplatin: the oxaliplatin-resistant colon cancer cells exhibited significantly downregulated miR-409-3p levels, but higher autophagic activity than the oxaliplatinsensitive cells. Using bioinformatics analysis, we predicted that miR-409-3p miRNA binds to the key autophagy gene encoding Beclin-1. Our findings indicated that the overexpression of miR-409-3p inhibited Beclin-1 expression and autophagic activity by binding to the 3'-untranslated region of Beclin-1 mRNA. In addition, the overexpression of miR-409-3p enhanced the chemosensitivity of the oxaliplatin-sensitive and oxaliplatinresistant colon cancer cells. The restoration of Beclin-1 abrogated these effects of miR-409-3p. In a xenograft model using nude mice, we examined the effects of miR-409-3p on tumor growth during chemotherapy. miR-409-3p overexpression sensitized the
\end{abstract}

Correspondence to: Professor Xiangliang Zhang, Department of Abdominal Surgery (Section 2), Affiliated Cancer Hospital of Guangzhou Medical University, 78 Hengzhigang Road, Guangzhou, Guangdong 510095, P.R. China

E-mail: zhx17229@163.com

*Contributed equally

Abbreviations: LC3, microtubule-associated protein light chain 3; miRNA or miR, microRNA; MTT, 3-(4,5-dimethylthiazol-2-yl)-2,5diphenyltetrazolium bromide; TUNEL, terminal deoxynucleotidyl transferase dUTP nick-end labeling; UTR, untranslated region

Key words: autophagy, chemoresistance, colon cancer, microRNA tumor to chemotherapy, while inhibiting chemotherapy-induced autophagy in a manner dependent on Beclin-1. The findings of our study suggest that miR-409-3p is capable of enhancing the chemosensitivity of colon cancer cells by inhibiting Beclin-1mediated autophagy.

\section{Introduction}

Colorectal cancer is the third most frequent cause of mortality in males and females, when the genders are considered separately and the second most frequent cause when they are considered together. In fact, colorectal cancer-related mortality accounts for approximately $10 \%$ of all cancer-related deaths (1). Although surgical resection is considered the most effective and curative treatment for various types of cancer, the majority of patients with colon cancer require neoadjuvant or adjuvant chemotherapy before and after surgery. Thus, chemotherapy is the most widely used form of treatment for colon cancer, whether used as monotherapy or as adjuvant therapy (2-4).

Colon cancer cells often become resistant during chemotherapy, which limits treatment efficacy and worsens patient prognosis $(5,6)$. Researchers are actively engaged in elucidating the mechanisms responsible for resistance to treatment in aim to identify and develop strategies to overcome it, not only in colon cancer, but also in other types of malignant disease (7).

A major mechanism of cancer cell chemoresistance is thought to be autophagy (8). Autophagy is an otherwise normal process in which dysfunctional cytoplasmic proteins and organelles are sequestered in intracellular autophagosomes that fuse with lysosomes and are degraded. Autophagy normally occurs as an adaptive response to cellular stresses, such as nutrient starvation or metabolic stress. Autophagosome formation is triggered by class III phosphoinositide 3-kinase and Beclin-1, also known as the mammalian homologue of yeast autophagy-related gene 6 (9). Consistent with its critical role in autophagosome formation, Beclin-1 expression increases during autophagy (10). During this process, microtubule-associated protein light chain 3 (LC3) is converted from the cytosolic form, LC3-I, to the autophagic membranebound form, LC3-II; relative amounts of LC3-I and II serve as an index of autophagy (11). Autophagy is thought to promote chemoresistance by promoting cellular energy production. 
Consistent with this hypothesis, the inhibition of autophagy using 3-methyladenine and chloroquine has been shown to enhance the chemosensitivity of hepatocellular carcinoma and supraglottic laryngeal cancer cells (12-14). These data suggest that the inhibition of autophagy may reduce the risk or level of chemoresistance in colon cancer cells.

It may be possible to regulate autophagy by manipulating the levels of microRNAs (miRNAs or miRs), since these post-transcriptional regulators help to control numerous cellular processes (15). These short RNAs, 18-24 nt in length, bind to the 3'-untranslated region (UTR) of target mRNAs, destabilizing them and thereby inhibiting protein translation (16). Several miRNAs have been associated with tumorigenesis, response to treatment and patient survival (17-20). However, whether miRNAs play a role in the chemoresistance of cancer cells, including colon cancer cells, remains largely unknown.

The miRNA miR-409-3p has been shown to be significantly downregulated in fibrosarcoma, bladder and gastric cancer (21-23). Thus, we wished to determine whether miR-409-3p plays a role in chemoresistance in colon cancer. In the present study, we demonstrate firstly using bioinformatics analysis, and then using a dual-luciferase assay in LoVo human colon cancer cells, that miR-409-3p binds to the 3'-UTR of Beclin-1 mRNA and downregulates its expression. We also demonstrate that miR-409-3p expression is downregulated, whereas Beclin-1 expression and autophagic activity are markedly upregulated, in chemoresistant LoVo cell cultures. The overexpression of miR-409-3p in the same cell line or treatment of the cells with miR-409-3p mimic in the medium, significantly suppressed Beclin-1 expression and autophagic activity, which rendered the cell lines sensitive to oxaliplatin therapy. We concluded that increasing miR-409-3p expression may inhibit chemotherapy-induced autophagy and thereby provide a mechanism through which to enhance the sensitivity of colon cancer cells to chemotherapy.

\section{Materials and methods}

Cell culture, human tissue samples and mice. The human 293 cells, the human colon cancer cell lines (LoVo, HCT 116, DLD-1, SW480, HT-29 and RKO) and normal colon epithelial cell lines (FHC and CCD-18Co) were obtained from the Chinese Academy of Sciences (Shanghai, China) and cultured in Dulbecco's modified Eagle's medium (DMEM) supplemented with $10 \%$ fetal bovine serum (FBS) (both from Invitrogen, Carlsbad, CA, USA) containing penicillin/streptomycin. The cells were grown in a humidified $5 \% \mathrm{CO}_{2}$ atmosphere at $37^{\circ} \mathrm{C}$ in an incubator. The LoVo cell cultures were exposed to chemotherapy with oxaliplatin as previously described (24), at the Cancer Research Institute of Guangzhou Medical University (Guangzhou, China). The oxaliplatin-resistant cell line was established from LoVo parental cells by gradually treating the cells with increasing concentrations of oxaliplatin for 3 months for the selection of oxaliplatin-resistant cells. The cells were subcultured at $10 \%$ confluency by splitting at a ratio of 1:200 and exposed to increasing concentrations of oxaliplatin $(0,50$, 100 and $200 \mu \mathrm{g} / \mathrm{ml}$ ). The cultures that survived after 8 cycles of subculturing and oxaliplatin treatment (maximum treatment, $200 \mu \mathrm{g} / \mathrm{ml}$ ) were considered chemoresistant and used in the subsequent experiments. It should be noted that the LoVo cells were selected as the target cells for use in the experiments, as they were more invasive than the other cells (data not shown). The $\mathrm{IC}_{50}$ value was read from the drug concentration which had the half maximal inhibitory effect on cell viability. In addition, the accumulation of autophagosomes in the colon cancer cells was measured using an electron microscope (Philips CM-120 transmission electron microscope; Philips, Amsterdam, The Netherlands) as previously described (12). Autophagosomes appear in cells as single membrane vesicles containing different cytoplasmatic material.

A total of 30 human samples were used in this study, including 20 human colon tumor tissue samples and 10 human normal colon tissue (adjacent) samples. The samples were obtained from patients undergoing surgery for colon cancer between 2013 and 2014 at the Department of Abdominal Surgery, The Affiliated Cancer Hospital of Guangzhou Medical University (Guangzhou, China) and stored in liquid nitrogen until analysis. The tumor and normal samples were obtained following a protocol approved by the Ethics Committee of the Department of Guangzhou Medical University, The Affiliated Cancer Hospital (Guangzhou, China). All patients provided written informed consent for the use of their samples for experimental purposes.

Female BALB/c nude mice $(n=20,6$ weeks old, weighing 25-30 g) were obtained from the Medical Experimental Animal Center of Guangdong Province (Guangzhou, China) and housed under pathogen-free conditions with free access to food and water. The animal experimental procedures were reviewed and approved by the Institutional Animal Care and Use Committee of Guangzhou Medical University.

Determination of the interaction between miR-409-3p and Beclin-1 3'-UTR using a Dual-Luciferase Reporter assay. The wild-type (WT) 3'-UTR of human Beclin-1 (5'-AGGTTGAG AAAGGCGAGACA-3') and the 3'-UTR sequences carrying mutations (C5878T, A5999T or A6328G) were amplified and subcloned into the pGL3 expression vector downstream of the promoter and coding region of firefly luciferase (Promega Corp., Madison, WI, USA); subcloning was performed using the $\mathrm{XbaI}$ and Not I restriction sites. The resulting expression plasmids were transfected into the chemoresistant LoVo cells in the presence or absence of $20 \mathrm{nmol} / \mathrm{ml} \mathrm{miR}-409-3 \mathrm{p}$ mimic (Gene Pharma, Shanghai, China) using Lipofectamine transfection reagent (Invitrogen) according to the manufacturer's instructions. The cells were harvested $48 \mathrm{~h}$ after transfection and the luciferase activity was measured using a Dual-Luciferase Reporter assay kit (Promega Corp.). Luciferase activity was normalized to that measured in the control cells transfected with the empty pGL3 expression vector in the presence or absence of miR-409-3p mimic. In addition TargetScan was used to confirm the target of miR-409-3p.

Cell viability and colony formation assays. The chemoresistant LoVo cells were pre-treated with miR-409-3p mimic or scrambled miRNA (5'-ATTAATCATAGAGGAAATCCACG-3'; Shanghai Kangcheng Biological engineering Co. Ltd. Shanghai, China) for $1 \mathrm{~h}$ and then exposed to oxaliplatin at $50 \mu \mathrm{g} / \mathrm{ml}$ for $24 \mathrm{~h}$. Cell growth and viability were evaluated by 3-(4,5-dimethylthiazol-2-yl)-2,5-diphenyltetrazolium bromide (MTT) assay (Sangon Biotech, Shanghai, China). 
Briefly, the cells were seeded in 96-well plates at a density of $5 \times 10^{3}$ cells $/ 200 \mu \mathrm{l}$. Following transfection and chemotherapy, fresh medium containing MTT solution [5 $\mathrm{mg} / \mathrm{ml}$ diluted in phosphate-buffered saline (PBS), $20 \mu \mathrm{l} /$ well] was added followed by incubation for an additional $4 \mathrm{~h}$. The resulting formazan was resuspended in dimethyl sulfoxide (200 $\mu \mathrm{l} /$ well). Finally, the absorbance was determined at $490 \mathrm{~nm}$ using an ELISA reader (BioTek Instruments, Inc., Winooski, VT, USA).

The colony-forming ability was assessed at 15 days following transfection. The medium was discarded and the cell colonies were stained with crystal violet (Sigma-Aldrich (Shanghai, China) prior to counting using the Leica Zoom 2000 dissecting microscope (Leica Microsystems Inc., Buffalo Grove, IL, USA). Measurements were taken in quadruplicate from 3 independent experiments.

miRNA or plasmid DNA transfection. miRNA mimics and control miRNAs were purchased from Applied Biosystems Life Technologies (Foster City, CA, USA). Lipofectamine 2000 (Invitrogen) was used for the transfection of miRNA mimics or plasmid DNA according to the manufacturer's instructions. At $48 \mathrm{~h}$ after transfection, the expression of miR-409-3p was detected by reverse transcription quantitative-polymerase chain reaction (RT-qPCR).

Cell viability assay. A total of $1 \times 10^{4}$ cells per well were seeded in 48-well plates overnight. The medium was replaced with fresh medium with or without oxaliplatin at the indicated concentrations and incubated for $48 \mathrm{~h}$. Cell viability was measured by MTT assay. The absorbance was measured spectrophotometrically at $570 \mathrm{~nm}$ using the Universal Microplate Reader EL800 (BioTek Instruments, Inc.).

$R T-q P C R$. Total RNA was isolated from the cells using TRIzol reagent (Invitrogen) and small RNA was extracted using the mirVana kit (Ambion, Austin, TX, USA) according to the manufacturer's instructions. The corresponding cDNA was generated using M-MLV reverse transcriptase (Clontech Laboratories, Palo Alto, CA, USA) and the TaqMan microRNA reverse transcription kit (Applied Biosystems, Foster City, CA, USA). The RT-qPCR mixtures, which contained cDNA templates, primers and SYBR-Green qPCR Master Mix, were subjected to RT-qPCR quantification, and Beclin-1 and miR-409-3p expression levels were quantified using the $2^{-\Delta \Delta C}$ method. The expression levels were expressed relative to the levels of the following internal control genes: glyceraldehyde3-phosphate dehydrogenase (GAPDH) for Beclin-1 or U6 small nuclear (sn)RNA for miR-409-3p.

Western blot analysis. Total cell lysates were separated by $10 \%$ sodium dodecyl sulfate-polyacrylamide gel electrophoresis and transferred onto nitrocellulose membranes (Amersham; GE Healthcare, Little Chalfont, UK). Non-fat dry milk $(2.5 \%)$ was used to block the membranes, which were then incubated with primary antibody against LC3 (ab63817) and Beclin-1 (ab62557) (both from Abcam, Cambridge, UK) or cleaved caspase-3 (PC679-50UG; Millipore Corp., Boston, MA, USA) and anti-GAPDH antibody (bs-2188R; BIOSS, Beijing, China). The membranes were then incubated with horseradish peroxide (HRP)-conjugated antibody and antibody binding was visualized using enhanced chemiluminescence (ECL; Amersham; GE Healthcare). Band intensities were quantitated using Image Pro Plus 6.0 (Olympus, Osaka, Japan) and normalized to the levels of GAPDH in the same sample.

Tumorigenicity assay. Chemoresistant LoVo cells $\left(2 \times 10^{6}\right)$ diluted in $200 \mu \mathrm{l}$ PBS pre-transfected with lentiviral vector expressing miR-409-3p or scrambled miRNA control were injected subcutaneously into the right groin of the BALB/c nude mice. Tumor length and width were measured daily and the volume was calculated using the following formula: length $\mathrm{x}$ width ${ }^{2} \mathrm{x} \pi / 6$. When the tumor volume reached approximately $500 \mathrm{~mm}^{3}$, the tumor was injected once daily with oxaliplatin $(50 \mu \mathrm{g} / \mathrm{ml}, 1 \mathrm{ml})$ or PBS as a control and the volume was measured daily for at least 36 more days. On day 41, the animals were euthanized by cervical dislocation following exposure to $\mathrm{CO}_{2}$. The tumors were removed and either frozen in liquid nitrogen or fixed in $10 \%$ formalin for further analysis.

Statistical analysis. Data are expressed as the means \pm standard deviation (SD) of 3 independent experiments. Differences between or within groups were assessed for significance using, respectively, Student's two-tailed t-test or one-way ANOVA. The threshold for significance was defined as $\mathrm{P}<0.05$.

\section{Results}

miR-409-3p is downregulated in human colon tumors and suppresses colon cancer cell proliferation in vitro. Previous studies have indicated that miR-409-3p acts as a tumor suppressor in various types of cancer (21-23). To examine the role of miR-409-3p in human colon cancer, we measured the expression of miR-409-3p in colon cancer and normal colon cell lines. As expected, the miR-409-3p levels were significantly downregulated in all the colon cancer cell lines compared with the 2 normal cell lines, FHC and CCD-18Co (Fig. 1A). To support these findings, we compared the miR-409-3p levels between the human colon cancer samples and the adjacent benign tissue samples. Consistent with these results, miR-409-3p expression was downregulated in the colon cancer samples, compared with the normal colon tissue samples (Fig. 1B), thus suggesting that miR-409-3p acts as a tumor suppressor in colon cancer. We then transfected miR-409-3p mimics or control miRNA into the LoVo colon cancer cells. Our results revealed that cell proliferation was suppressed by the overexpression of miR-409-3p (Fig. 1C). Cell proliferation at 48, 72 and $96 \mathrm{~h}$ was significantly suppressed following transfection with miR-409-3p mimic. Moreover, we observed that miR-409-3p overexpression in the LoVo cells significantly inhibited the colony-forming ability of th cells (Fig. 1D). Taken together, these results revealed a tumor suppressor role for of miR-409-3p in colon cancer.

miR-409-3p epxression negatively correlates with resistance to oxaliplatin in colon cancer cells. The evasion of chemotherapeutic agents has been recognized as one of the hallmarks of cancer (3). In this study, to investigate the putative roles of miR-409-3p in enhancing the chemosensitivity of colon cancer cells, we established the oxaliplatin-resistant cell line from LoVo parental cells by gradually treating the parental cells 
A

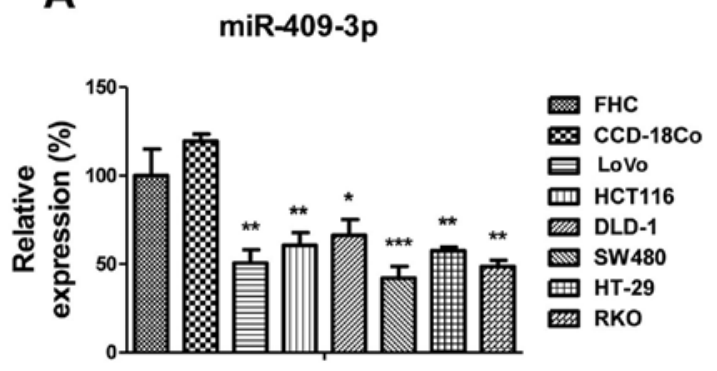

C

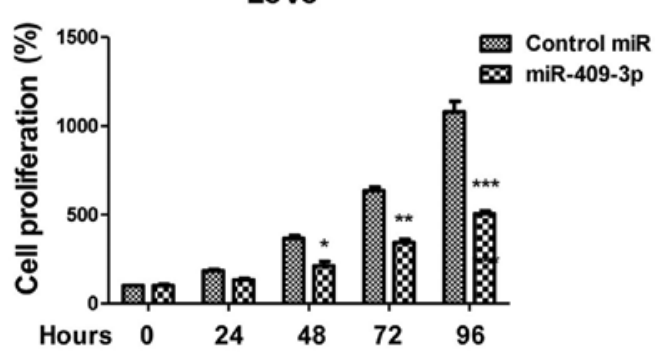

B

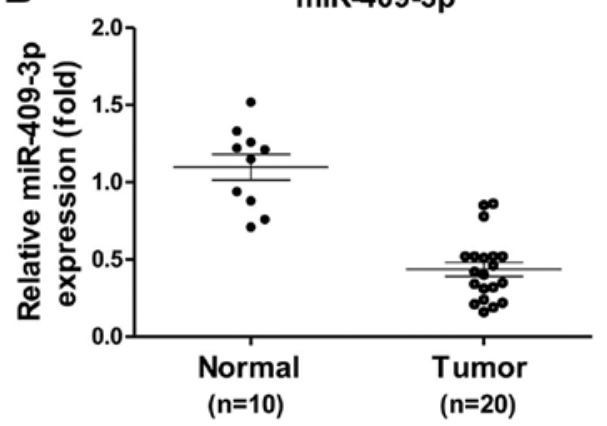

LoVo

D

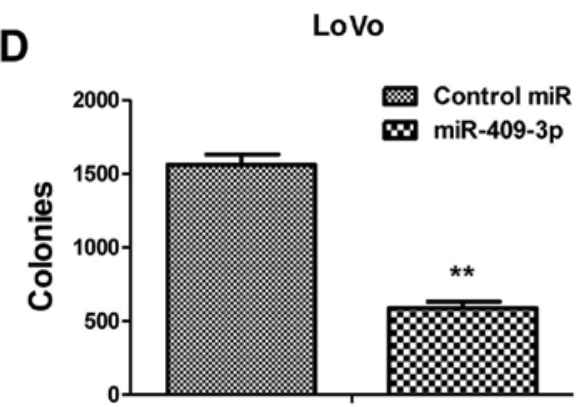

Figure 1. Tumor suppressor roles of miR-409-3p in human colon cancers cells. (A) The expression of miR-409-3p in human normal colon epithelial cell lines (FHC and CCD-18Co) and human colon cancer cell lines (LoVo, HCT 116, DLD-1, SW480, HT-29 and RKO). (B) The expression of miR-409-3p in human colon tumor samples and adjacent normal colon tissues. (C) LoVo cells were transfected with control miRNA or miR-409-3p mimic for $48 \mathrm{~h}$, followed by the measurement of cell proliferation rates. (D) LoVo cells were transfected with control miRNA or miR-409-3p mimic for $48 \mathrm{~h}$, the cells were digested and re-plated in regular cell culture conditions, followed by colony formation assay.
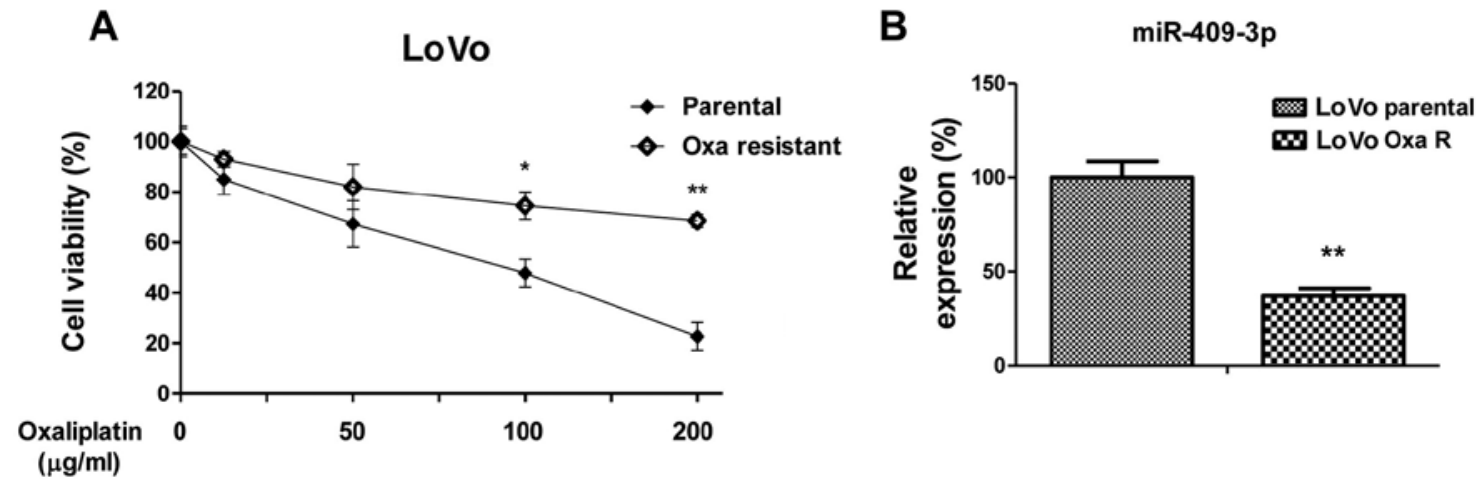

Figure 2. miR-409-3p expression negatively correlates with oxaliplatin sensitivity. (A) Generation of oxaliplatin-resistant (Oxa R) cells from LoVo cells. Parental cells were treated with gradually increasing concentrations of oxaliplatin in regular cell culture conditions to select resistant cells. Oxaliplatin-resistant LoVo clones were pooled and treated with oxaliplatin at the indicated concentrations for $48 \mathrm{~h}$. (B) The expression of miR-409-3p in the LoVo parental cells and oxaliplatin-resistant cells. Columns, mean of 3 independent experiments; bars represent the means $\pm \mathrm{SD}$. ${ }^{*} \mathrm{P}<0.05$ and ${ }^{* *} \mathrm{P}<0.01$.

with increasing concentrations of oxaliplatin for 3 months to select oxaliplatin-resistant cells. Fig. $2 \mathrm{~A}$ illustrates that the $\mathrm{IC}_{50}$ value of the oxaliplatin-resistant cells was approximately 5-fold greater than the $\mathrm{IC}_{50}$ value of the LoVo parental cells, indicating that the resistant cells may be tolerant to higher concentrations of oxaliplatin. Of note, miR-409-3p expression was significantly lower in the oxaliplatin-resistant cultures than in the parental cells (Fig. 2B), indicating that miR-409-3p may be a therapeutic target for the treatment of chemoresistant cancer cells.

Oxaliplatin-resistant cancer cells exhibit elevated autophagic activity. The above-mentioned findings demonstrated the nega- tive correlation between the expression of miR-409-3p and sensitivity to oxaliplatin in colon cancer cells. To elucidate the mechanisms responsible for the miR-409-3p-mediated chemosensitivity, we compared the expression levels of Beclin-1 and its downstream factors, LC3-I and LC3-II between the parental and oxaliplatin-resistant LoVo cells. As expected, western blot analysis revealed that Beclin-1 and LC3-II protein expression was present at much higher levels and that LC3-I protein expression was downregulated in the chemoresistant cells compared with the parental cells, indicating enhanced autophagic activity in the oxaliplatin-resistant cells (Fig. 3A). Consistent with these findings, the mRNA expression levels of LC3-I were downregulated and those of LC3-II were 
A

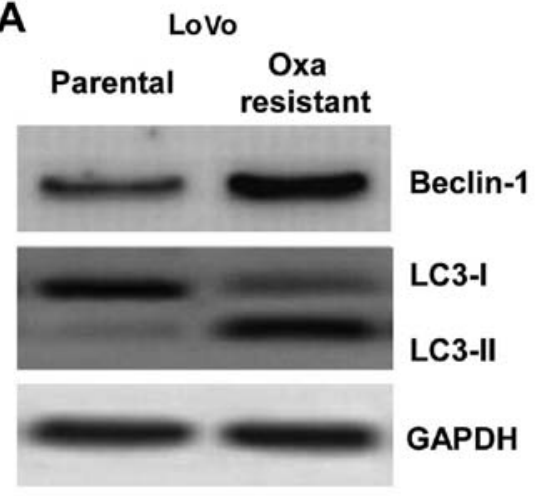

B
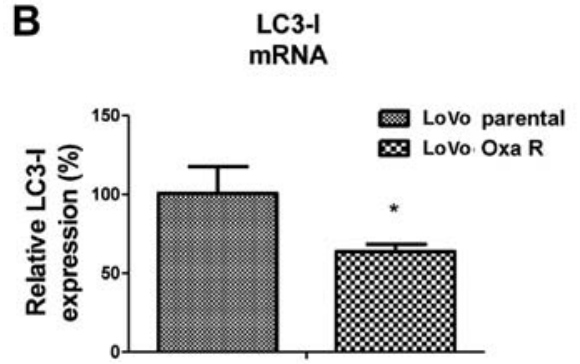

C

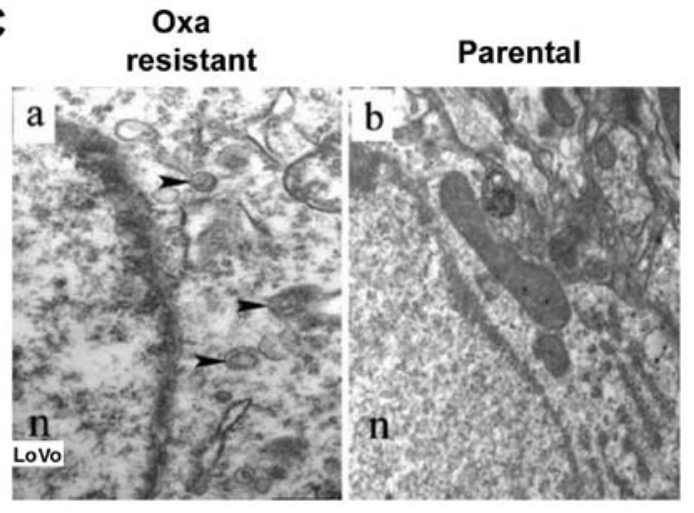

LC3-II

mRNA

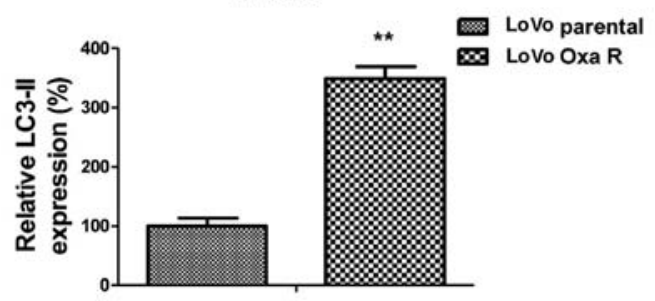

Figure 3. Elevated Beclin-1 expression and autophagic activity in LoVo oxaliplatin-resistant (Oxa-R) cells. (A) Expression of Beclin-1, LC3-I and LC3-II was measured by western blot analysis in the oxaliplatin-sensitive and -resistant LoVo cells as indicators of autophagy levels in chemoresistant cells. GAPDH was used as a loading control. (B) mRNA expression of LC3-I/II in parental and chemoresistant cells measured by RT-qPCR. (C) Autophagosome formation in (panel a) chemoresistant and (panel b) chemosensitive LoVo cancer cells observed under an electron microscope (n, nucleus; x300,000 magnification). LC3-II dots are represented by black arrowheads, indicating the presence of autophagosomes. Columns represent the means of 3 independent experiments; bars represent the means $\pm \mathrm{SD}$. ${ }^{*} \mathrm{P}<0.05$ and ${ }^{* *} \mathrm{P}<0.01$.

upregulated in the chemoresistant cells (Fig. 3B). However, we did not observe a significant difference in Beclin-1 mRNA expression levels between the resistant and sensitive cells, indicating that the post-transcriptional regulation of Beclin-1 may occur in oxaliplatin-resistant cells (data not shown). To further confirm our findings, we measured autophagosome formation in the chemoresistant (Fig. 3C, panel a) and parental (Fig. 3C, panel b) LoVo cancer cells. The LC3-II dots (representing the autophagic activity; black arrowheads represent autophagosomes) in the oxaliplatin-resistant cells were more evident than in the sensitive cells (Fig. 3C). The above-mentioned data (Figs. 2 and 3) indicate that miR-409-3p expression is downregulated in chemotherapy-resistant colon cancer cells and that autophagy is activated in these resistant cells, thus suggesting a link between the downregulation of miR-409-3p expression and the activation of autophagy.

miR-409-3p directly targets Beclin-1 to regulate autophagic activity. To obtain more direct evidence that miR-409-3p regulates autophagy, we performed a screening of the possible binding sites of miR-409-3p in the 3'-UTRs of gene transcripts related to autophagy using TargetScan. This analysis highlighted Beclin-1 as a putative target of miR-409-3p. To verify that miR-409-3p targets Beclin-1, we measured the protein expression levels of Beclin-1 and LC3-I/II in the LoVo cells in response to $\mathrm{miR}-409-3 \mathrm{p}$ overexpression induced by transfection with miR-409-3p mimics. In the cells transfected with miR-409-3p mimics, we observed decreased Beclin-1 expression levels, as well as increased LC3-I levels and decreased LC3-II levels (Fig. 4A). To verify that miR-409-3p binds to the
3'-UTR of Beclin-1 directly, we transfected 293 cells with a dual-luciferase reporter system in which the luciferase transcript was fused to the 3'-UTR of Beclin-1. In this system, luciferase activity is lower in the presence of elements that bind to the UTR. The cells were transfected with either the reporter system alone or with the reporter system together with the miR-409-3p mimic. Other cells were co-transfected with a reporter system containing a mutated version of the Beclin-1 3'-UTR lacking the predicted miR-409-3p binding site. The relative luciferase activity was significantly lower in the cultures co-transfected with miR-409-3p mimic and pGL3 containing the WT 3'-UTR of Beclin-1 than in the cells co-transfected with the mimic and pGL3 containing the mutant 3'-UTR (Fig. 4B). These results confirmed that Beclin-1 is a target gene of miR-409-3p. Consistent with the results from western blot analysis, the mRNA levels of LC3-I and LC3-II were significantly altered by transfection with miR-409-3p (Fig. 4C). Taken together, the above-mentioned results demonstrate that miR-409-3p suppresses autophagy through the direct inhibition of Beclin-1.

Exogenous overexpression of miR-409-3p enhances the sensitivity of oxaliplatin-resistant and non-resistant colon cancer cells through the inhibition of Beclin-1. To determine the effects of miR-409-3p on the drug sensitivity of colon cancer cells, we pre-treated the LoVo cells with miR-409-3p mimic or scrambled miRNA control and measured the cell viabilitys in response to treatment with oxaliplatin. Exogenous miR-409-3p overexpression led to significantly higher apoptotic levels (Fig. 5A, shown by a decrease in the number of surviving cells). These results suggest that miR-409-3p enhances 
A

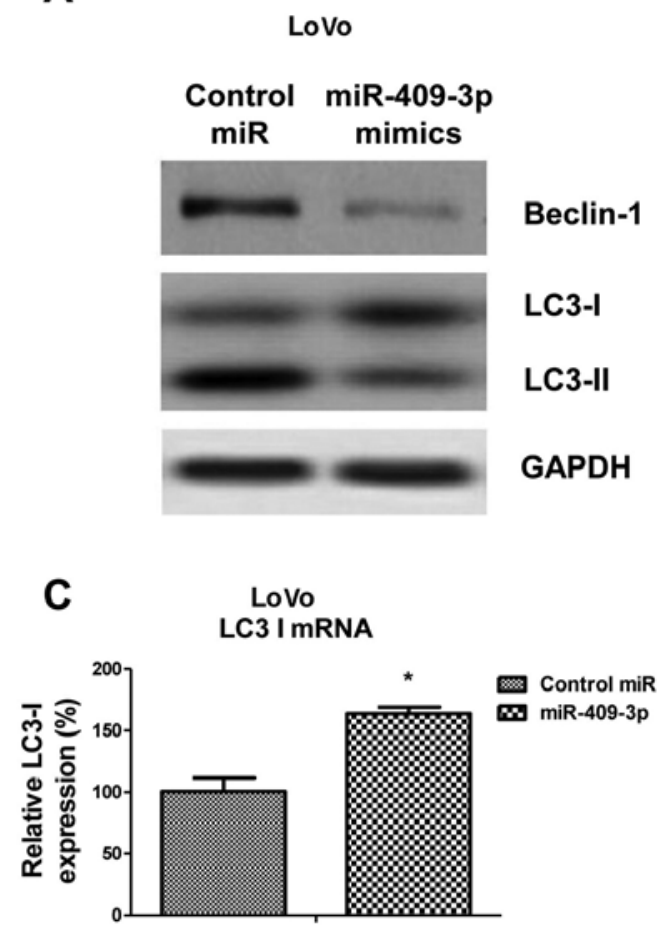

B 5'-uugagaca gcuaagcuuagcuucagu-3' Beclin-1 3'-UTR

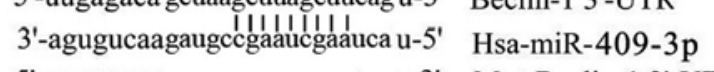

5'-uugagaca auuaacaguacacucguu-3' Mut-Beclin-1 3'-UTR
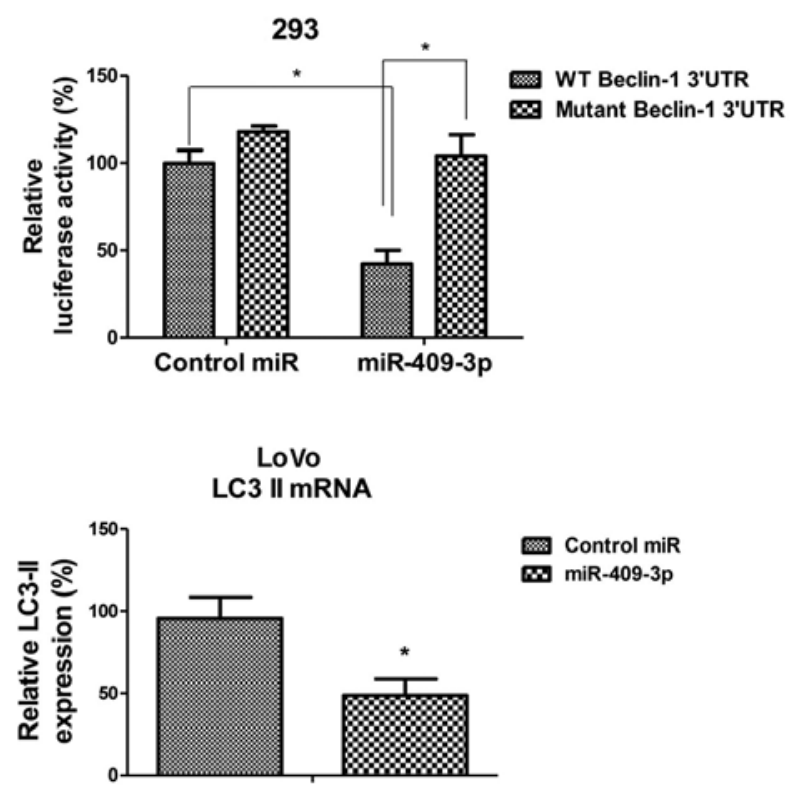

Figure 4. miR-409-3p directly targets Beclin-1 through specific binding to the 3'-UTR of Beclin-1 mRNA. (A) LoVo cells were transfected with control miRNA or miR-409-3p mimic for $48 \mathrm{~h}$, and the expression levels of Beclin-1, LC3-I/II were then measured by western blot analysis. GAPDH was used as a loading control (B) Binding sequences for miR-409-3p in the Beclin-1 3'-UTR based on prediction (by TargetScan) and the mutations on the 3'-UTR of Beclin-1 are shown. DualLuciferase activity assays to detect the interaction between miR-409-3p and Beclin-1 3'-UTR. LoVo cells were transfected with luciferase constructs containing wild-type (WT) or mutant (Mut) Beclin-1 3'-UTR in the presence of either miR-409-3p mimic or scrambled miRNA control. Transfected cells were incubated for $48 \mathrm{~h}$ and processed using a Dual-Luciferase Reporter assay kit. Hsa, Homo sapiens; WT, pGL3 expression plasmid carrying the luciferase gene fused to the wild-type Beclin-1 3'-UTR; Mut, pG3 expression plasmid carrying the luciferase gene fused to mutated Beclin-1 3'-UTR. (C) LoVo cells were transfected with control miRNA or miR-409-3p mimic for $48 \mathrm{~h}$, and LC3-I-II mRNA levels were measured by RT-qPCR. Columns represent the means of 3 independent experiments; bars represent the means $\pm \mathrm{SD}$. ${ }^{*} \mathrm{P}<0.05$.
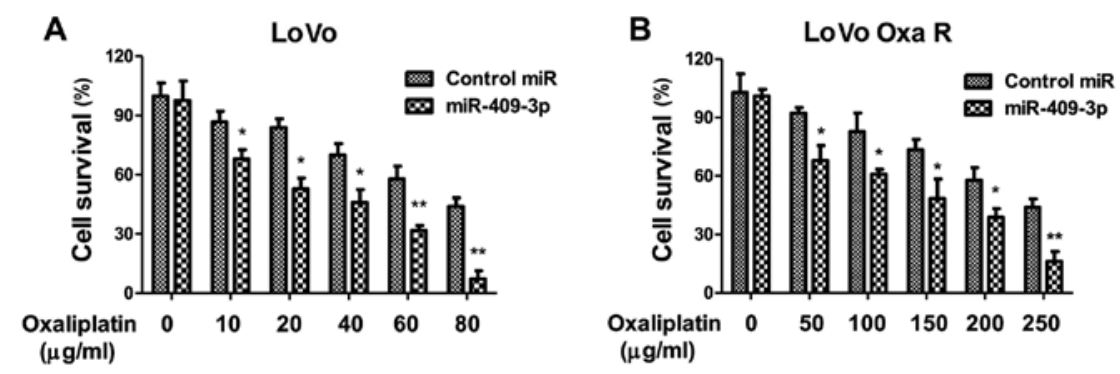

Figure 5. Effects of miR-409-3p on cell sensitivities to oxaliplatin in chemosensitive and -resistant LoVo cells. (A) LoVo cells and (B) Oxaliplatin-resistant LoVo cells (LoVo Oxa R) were transfected with control miRNA or miR-409-3p mimic for $48 \mathrm{~h}$, and the cells were then treated with oxaliplatin at the indicated concentrations for $48 \mathrm{~h}$, followed by the assessment of cell survival rates. (B) Lovo Oxaliplatin-resistant LoVo cells were transfected with control miRNA or miR-409-3p mimic for $48 \mathrm{~h}$, the cells were then treated with oxaliplatin at the indicated concentrations for $48 \mathrm{~h}$, followed by the assessment of cell survival rates. Columns represent the means of 3 independent experiments; bars represent the means $\pm \mathrm{SD}$. ${ }^{*} \mathrm{P}<0.05$ and ${ }^{* *} \mathrm{P}<0.01$.

the sensitivity of LoVo colon cance4r cells by triggering apoptosis. As expected, the overexpression of miR-409-3p in LoVo oxaliplatin-resistant LoVo cells sensitized the cells to oxaliplatin. The oxaliplatin-resistant cells transfected with control miRNA showed an $\mathrm{IC}_{50}$ value of $250 \mu \mathrm{g} / \mathrm{ml}$, which was significantly higher than that of the cells transfected with miR-409-3p mimic (Fig. 5B), indicating miR-409-3p may be a therapeutic agent which may be used to overcome resistance to oxaliplatin. To obtain additional evidence that miR-409-3p sensitizes chemoresistant LoVo cells to chemotherapy by regulating Beclin-1 expression, we co-transfected the cells with miR-409-3p mimic and an expression plasmid carrying the Beclin-1 coding sequence upstream of a 3'-UTR mutated to eliminate the miR-409-3p binding site. The overexpression of Beclin-1 (Fig. 6A) almost completely blocked the ability of miR-409-3p to inhibit the growth of chemoresistant LoVo cells, as well as the ability of miR-409-3p to trigger apoptosis (Fig. 6B). These results support the hypothesis that miR-409-3p sensitizes chemoresistant LoVo colon cancer cells to chemotherapy by targeting Beclin-1-mediated autophagy. 
A

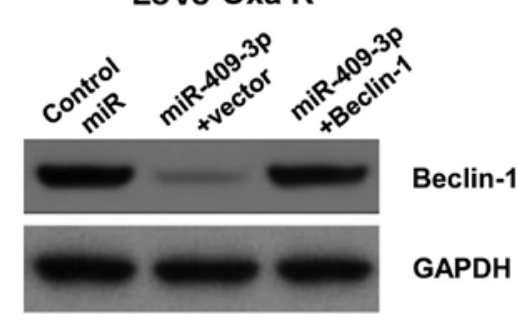

B

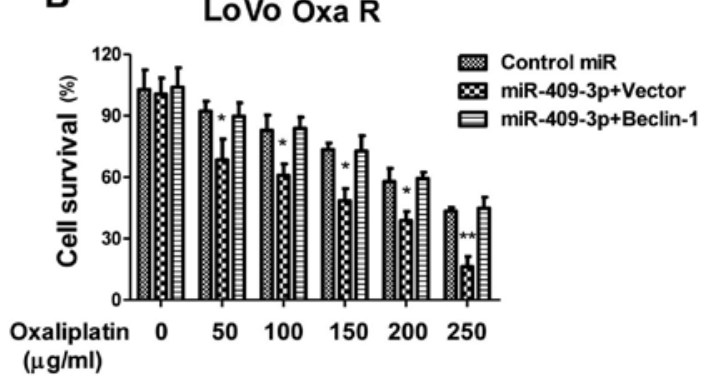

Figure 6. Effect of Beclin-1 overexpression on miR-409-3p-induced chemosensitivity in chemoresistant Lovo cells (LoVo Oxa R). Cells were transfected with miR-409-3p mimic in the presence or absence of Beclin-1 overexpression plasmid. Parallel control cultures were treated with scrambled miRNA instead of miR-409-3p mimic. (A) Western blot analysis of Beclin-1. GAPDH was a loading control. (B) Cell survival analysis following treatment with oxaliplatin by MTT assay. Columns represent the means of 3 independent experiments; bars represent the means $\pm \mathrm{SD}$. ${ }^{*} \mathrm{P}<0.05$ and ${ }^{* * *} \mathrm{P}<0.01$.

A

\section{Xenograft}

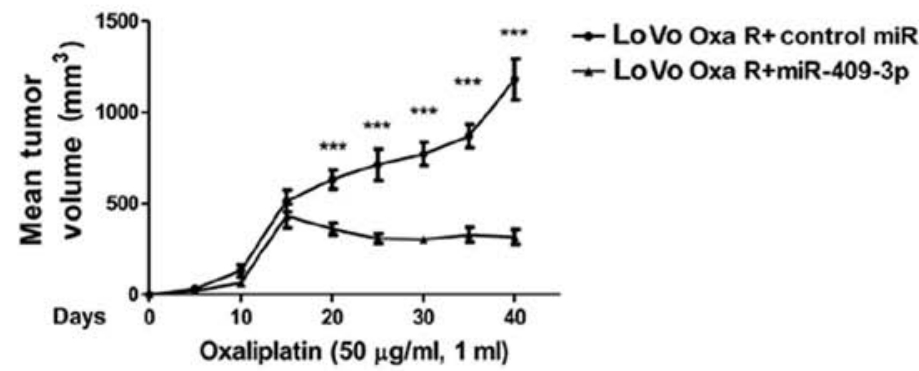

C

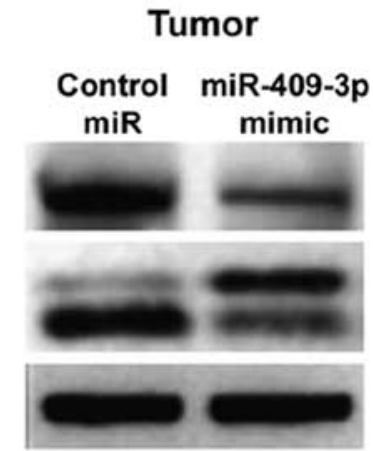

D

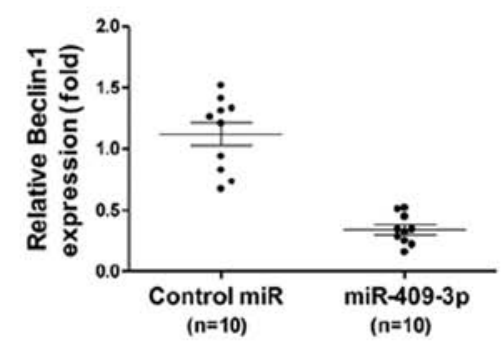

B

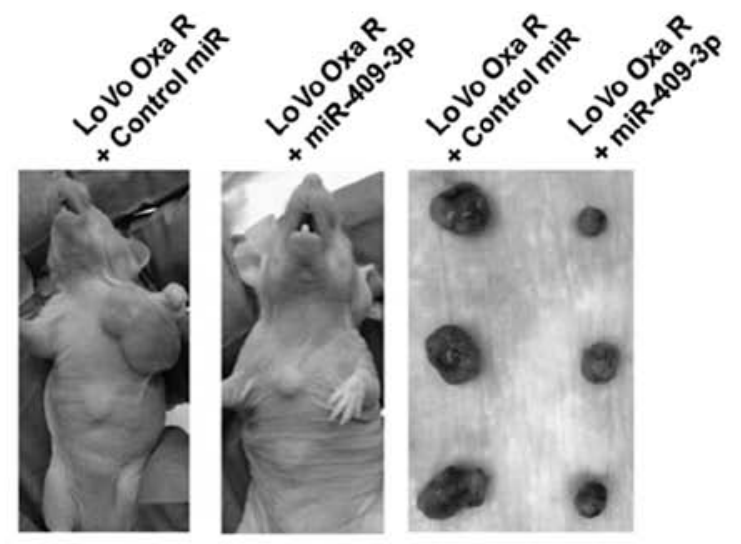

$\mathbf{E}$

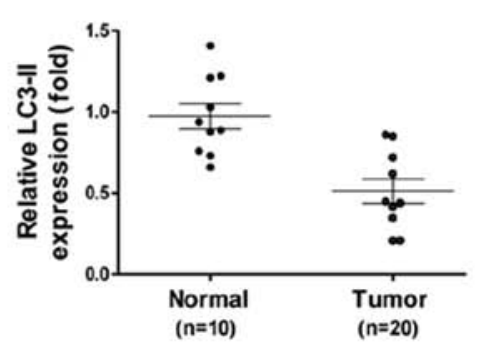

Figure 7. Effects of miR-409-3p on xenograft tumor growth in vivo. (A) Nude mice were subcutaneously injected with LoVo cells infected with lentivial (LV) plasmid expressing miR-409-3p or scrambled miRNA control. At 16 days after infection, when the average tumor volume had increased to approximately $500 \mathrm{~mm}^{3}$, the tumors were injected once with oxaliplatin $(50 \mu \mathrm{g} / \mathrm{ml}, 1 \mathrm{ml})$, and then the mean tumor volumes were measured twice a week. (B) The tumor volumes from oxaliplatin-treated mice with LV plasmid expressing miR-409-3p or scrambled miRNA control. (C) Western blot analysis of Beclin-1 and LC3-I/II protein levels in xenograft tumors. (D) The mRNA levels of Beclin-1 and (E) LC3-II were measured by RT-qPCR in xenograft tumors relative to GAPDH. Columns represent the means of 3 independent experiments; bars represent the means $\pm \mathrm{SD} .{ }^{* * * *} \mathrm{P}<0.001$.

Overexpression of miR-409-3p sensitizes oxaliplatin-resistant colon cancer cells by suppressing Beclin-1-mediated autophagy in vivo. To verify our in vitro experiments using the LoVo cell cultures, we determine whether miR-409-3p was capable of strengthening the ability of chemotherapeutic drugs to suppress the growth of tumor implanted tumors in nude mice. Twenty animals were equally divided into 2 groups, and subcutaneously injected with LoVo cells pre-transfected with lentiviral vector expressing miR-409-3p or scrambled miRNA control.
When the tumors attained a volume of approximately $500 \mathrm{~mm}^{3}$ (around day 16), the tumors were injected once daily with oxaliplatin $(50 \mu \mathrm{g} / \mathrm{ml}, 1 \mathrm{ml})$. The tumors overexpressing miR-409-3p were significantly more sensitive to chemotherapy than the tumors expressing the scrambled control (Fig. 7A and B). In addition, the analysis of the xenograft tumor samples at the end of chemotherapy indicated that the tumors overexpressing miR-409-3p expressed lower levels of Beclin-1 and exhibited less autophagic activity (Fig. 7C-E). These results are consis- 
tent with those from our in vitro experiments, suggesting that miR-409-3p sensitizes LoVo cancer cells to chemotherapy by blocking Beclin-1-mediated autophagy.

\section{Discussion}

In this study, using a combination of in vitro experiments using chemoresistant LoVo human colon cancer cells and in vivo experiments using a xenograft tumor model in nude mice, we provide evidence that miR-409-3p inhibits Beclin-1-mediated autophagy, thus rendering cancer cells sensitive to oxaliplatin therapy. Our results are consistent with growing evidence which suggests that autophagy plays a key role in the chemoresistance of cancer cells $(13,14)$, and our findings identify miR-409-3p as having potential therapeutic potential. Our results also support the hypothesis that miR-409-3p plays an important role in the onset and/or progression of colon cancer.

The role of miR-409-3p in cancer is complex. In prostate cancer, this miRNA appears to act as an oncogene, inhibiting the expression of the tumor suppressor genes, Ras suppressor 1 and stromal antigen 2 and thereby promoting tumorigenesis (25). In laryngeal cancer, miR-409-3p again appears to act as an oncogene, specifically inhibiting Krüppel-like factor 17 , E-cadherin and phosphatidylinositol 3-kinase and thereby promoting the epithelial-mesenchymal transition of tumor cells (26). Conversely, in gastric cancer, miR-409-3p appears to act as a tumor suppressor (23). The levels of this miRNA have been shown to be significantly lower in human colon cancer cells, than in normal human colon cells (27). In fact, miR-409-3p has been proposed to serve as a biomarker of gastric cancer (23). Moreover, miR-409-3p has been shown to inhibit HT1080 cell proliferation, vascularization and metastasis by targeting angiogenin (21). In the present study, we have provide evidence that miR-409-3p suppresses colon cancer cell proliferation, in part, by inhibiting autophagy mediated by Beclin-1.

Studies on various types of cancer point to autophagy as an important mechanism of cancer cell resistance to treatment. Gamma radiation has been shown to induce autophagy that renders glioma stem cells radioresistant $(28,29)$. It has also been shown that hypoxia-induced autophagy may contribute to the radioresistance of breast cancer cells (30). Hypoxiainducible factor (HIF)1- $\alpha$-mediated autophagy in cervical cancer cells may contribute to paclitaxel chemoresistance (31), while autophagy in LoVo colon cancer cells has been directly associated with resistance to irinotecan (32). The inhibition of autophagy in HCT 116 Bax-deficient colon cancer cells using the drug 3-methyladenine has been shown to enhance purvalanolinduced apoptotic cell death (33). The overexpression of miR-502, which inhibits Rab1B-mediated autophagy, has been demonstrated to inhibit the progression of HCT 116 and SW480 colon cancer cells in vitro and the growth of a tumor xenograft in vivo (34).

In this study, in continuation of these above-mentioned findings, we demonstrated that in oxaliplatin-resistant LoVo colon cancer cells, the miR-409-3p levels were significantly downregulated and that autophagic activity was increased. This suggests that miR-409-3p helps to regulate autophagy. Consistent with this possibility, our bioinformatics analysis predicted that the miRNA binds to the 3'-UTR of the key autophagy gene encoding Beclin-1, which we confirmed in
LoVo cells using a Dual-Luciferase Reporter assay. In addition, the experiments in which LoVo cells were co-transfected with the Beclin-1 expression plasmid and with a miR-409-3p mimic revealed that the antagonistic effects of the two molecules were lost when the putative miRNA binding sites in the 3'-UTR were mutated. It appears that miR-409-3p does for oxaliplatin therapy what miR-30a does for cis-dichloro-diamine platinum therapy: both miRNAs inhibit Beclin-1-mediated autophagy, thereby sensitizing cancer cells to chemotherapy (35). In lung cancer cells, the downregulation of miR-17-5p expression has been shown to lead to the upregulation of Beclin-1 expression and resistance to paclitaxel (36).

Taken together, our findings and those of previous studies highlight the possibility of targeting Beclin-1 as a therapeutic strategy for cancer. In fact, the levels of this protein have been shown to predict the overall survival of patients with esophageal squamous cell carcinoma following radiation therapy (37). However, it may not always be desirable to reduce Beclin-1 expression and therefore autophagy. While many studies point to autophagy as contributing to chemoresistance, other studies have reported the opposite. Akt inhibitors, for example, have been shown to promote radiosensitivity by inducing autophagy (38). Inhibitors of the mammalian target of rapamycin have been shown to radiosensitize non-small-cell lung cancer cells by inhibiting the activity of phosphatase and tensin homolog (PTEN) (39). Whether these opposite findings regardin the association between autophagy and resistance to treatment reflect differences between chemo- and radiotherapy and/or between different types of cancers warrant further investigation in future studies.

In conclusion, the present study provides evidence that miR-409-3p targets and inhibits Beclin-1, which inhibits chemotherapy-induced autophagy and thereby enhances the chemosensitivity of colon cancer cells.

\section{Acknowledgements}

The present study was supported by funding from the Guangdong Province Natural Science Fund (no. S2013010016662), the Health Bureau of Guangdong Province (nos. A2014224 and B2014196), the Science and Technology Planning Project of Guangdong Province (no. 2013B021800284) and the National Natural Science Foundation of China (nos. 81201932 and 81372493).

\section{References}

1. Tárraga López PJ, Albero JS and Rodríguez-Montes JA: Primary and secondary prevention of colorectal cancer. Clin Med Insights Gastroenterol 7: 33-46, 2014.

2. Bonetti A, Giuliani J and Muggia F: Targeted agents and oxaliplatin-containing regimens for the treatment of colon cancer. Anticancer Res 34: 423-434, 2014.

3. Gordeev SS, Rasulov AO, Mamedli ZZ, Aliev VA and Kulushev VM: Neoadjuvant chemotherapy for colorectal cancer - time to learn a new treatment strategy? Vopr Onkol 60: 18-24, 2014 (In Russian).

4. Walker AS, Zwintscher NP, Johnson EK, Maykel JA, Stojadinovic A, Nissan A, Avital I, Brücher BL and Steele SR: Future directions for monitoring treatment response in colorectal cancer. J Cancer 5: 44-57, 2014.

5. Chen J, Wang W, Zhang Y, Chen Y and Hu T: Predicting distant metastasis and chemoresistance using plasma miRNAs. Med Oncol 31: 799, 2014 
6. Touil Y, Igoudjil W, Corvaisier M, Dessein AF, Vandomme J, Monté D, Stechly L, Skrypek N, Langlois C, Grard G, et al: Colon cancer cells escape 5FU chemotherapy-induced cell death by entering stemness and quiescence associated with the c-Yes/YAP axis. Clin Cancer Res 20: 837-846, 2014.

7. Herraez E, Gonzalez-Sanchez E, Vaquero J, Romero MR, Serrano MA, Marin JJ and Briz O: Cisplatin-induced chemoresistance in colon cancer cells involves FXR-dependent and FXR-independent up-regulation of ABC proteins. Mol Pharm 9: 2565-2576, 2012.

8. Zhou Y, Sun K, Ma Y, Yang H, Zhang Y, Kong X and Wei L: Autophagy inhibits chemotherapy-induced apoptosis through downregulating Bad and Bim in hepatocellular carcinoma cells. Sci Rep 4: 5382, 2014

9. Helgason GV, Holyoake TL and Ryan KM: Role of autophagy in cancer prevention, development and therapy. Essays Biochem 55 133-151, 2013.

10. Maiuri MC, Zalckvar E, Kimchi A and Kroemer G: Self-eating and self-killing: crosstalk between autophagy and apoptosis. Nat Rev Mol Cell Biol 8: 741-752, 2007.

11. Mizushima N, Ohsumi Y and Yoshimori T: Autophagosome formation in mammalian cells. Cell Struct Funct 27: 421-429, 2002.

12. Song J, Guo X, Xie X, Zhao X, Li D, Deng W, Song Y, Shen F, $\mathrm{Wu} \mathrm{M}$ and Wei L: Autophagy in hypoxia protects cancer cells against apoptosis induced by nutrient deprivation through a Beclin1-dependent way in hepatocellular carcinoma. J Cell Biochem 112: 3406-3420, 2011.

13. Song J, Qu Z, Guo X, Zhao Q, Zhao X, Gao L, Sun K, Shen F, Wu M and Wei L: Hypoxia-induced autophagy contributes to the chemoresistance of hepatocellular carcinoma cells. Autophagy 5: 1131-1144, 2009

14. Kang R, Wang ZH, Wang BQ, Zhang CM, Gao W, Feng Y, Bai T, Zhang HL, Huang-Pu H and Wen SX: Inhibition of autophagypotentiated chemosensitivity to cisplatin in laryngeal cancer Hep-2 cells. Am J Otolaryngol 33: 678-684, 2012.

15. Mendell JT and Olson EN: MicroRNAs in stress signaling and human disease. Cell 148: 1172-1187, 2012.

16. Bartel DP: MicroRNAs: genomics, biogenesis, mechanism, and function. Cell 116: 281-297, 2004.

17. Oh JS, Kim JJ, Byun JY and Kim IA: Lin28-let7 modulates radiosensitivity of human cancer cells with activation of K-Ras. Int J Radiat Oncol Biol Phys 76: 5-8, 2010.

18. Wang P, Chen L, Zhang J, Chen H, Fan J, Wang K, Luo J, Chen Z, Meng Z and Liu L: Methylation-mediated silencing of the miR-124 genes facilitates pancreatic cancer progression and metastasis by targeting Racl. Oncogene 33: 514-524, 2014.

19. Wang P, Zhuang L, Zhang J, Fan J, Luo J, Chen H, Wang K, Liu L, Chen Z and Meng Z: The serum miR-21 level serves as a predictor for the chemosensitivity of advanced pancreatic cancer, and miR-21 expression confers chemoresistance by targeting FasL. Mol Oncol 7: 334-345, 2013.

20. Winter J, Jung S, Keller S, Gregory RI and Diederichs S: Many roads to maturity: microRNA biogenesis pathways and their regulation. Nat Cell Biol 11: 228-234, 2009.

21. Weng C, Dong H, Chen G, Zhai Y, Bai R, Hu H, Lu L and Xu Z: miR-409-3p inhibits HT1080 cell proliferation, vascularization and metastasis by targeting angiogenin. Cancer Lett 323: 171-179, 2012

22. Xu X, Chen H, Lin Y, Hu Z, Mao Y, Wu J, Xu X, Zhu Y, Li S, Zheng $X$ and Xie L: MicroRNA-409-3p inhibits migration and invasion of bladder cancer cells via targeting c-Met. Mol Cells 36: 62-68, 2013

23. Zheng B, Liang L, Huang S, Zha R, Liu L, Jia D, Tian Q, Wang Q, Wang C, Long Z, et al: MicroRNA-409 suppresses tumour cell invasion and metastasis by directly targeting radixin in gastric cancers. Oncogene 31: 4509-4516, 2012.
24. Zhang XL,Hu AB, Cui SZ and Wei HB: Thermotherapy enhances oxaliplatin-induced cytotoxicity in human colon carcinoma cells. World J Gastroenterol 18: 646-653, 2012.

25. Josson S, Gururajan M, Sung SY, Hu P, Shao C, Zhau HE, Liu C, Lichterman J, Duan P, Li Q, et al: Stromal fibroblast-derived miR-409 promotes epithelial-to-mesenchymal transition and prostate tumorigenesis. Oncogene 34: 2690-2699, 2014.

26. Tai J, Xiao X, Huang ZG, Yu ZK, Chen XH, Zhou WG, Chen XJ, Rao YS, Fang JG and Ni X: MicroRNAs regulate epithelialmesenchymal transition of supraglottic laryngeal cancer. Zhonghua Er Bi Yan Hou Tou Jing Wai Ke Za Zhi 48: 499-503, 2013 (In Chinese).

27. Liu M, Xu A, Yuan X, Zhang Q, Fang T, Wang W and Li C: Downregulation of microRNA-409-3p promotes aggressiveness and metastasis in colorectal cancer: an indication for personalized medicine. J Transl Med 13: 195, 2015.

28. Lomonaco SL, Finniss S, Xiang C, Decarvalho A, Umansky F, Kalkanis SN, Mikkelsen T and Brodie C: The induction of autophagy by $\gamma$-radiation contributes to the radioresistance of glioma stem cells. Int J Cancer 125: 717-722, 2009

29. Zhuang W, Qin Z and Liang Z: The role of autophagy in sensitizing malignant glioma cells to radiation therapy. Acta Biochim Biophys Sin (Shanghai) 41: 341-351, 2009.

30. He WS, Dai XF, Jin M, Liu CW and Rent JH: Hypoxia-induced autophagy confers resistance of breast cancer cells to ionizing radiation. Oncol Res 20: 251-258, 2012.

31. Peng X, Gong F, Chen Y, Jiang Y, Liu J, Yu M, Zhang S, Wang M, Xiao $\mathrm{G}$ and Liao $\mathrm{H}$ : Autophagy promotes paclitaxel resistance of cervical cancer cells: involvement of Warburg effect activated hypoxia-induced factor $1-\alpha$-mediated signaling. Cell Death Dis 5: e1367, 2014.

32. Chen MC, Lee NH, Ho TJ, Hsu HH, Kuo CH, Kuo WW, Lin YM, Tsai FJ, Tsai $\mathrm{CH}$ and Huang CY: Resistance to irinotecan (CPT-11) activates epidermal growth factor receptor/nuclear factor kappa B and increases cellular metastasis and autophagy in LoVo colon cancer cells. Cancer Lett 349: 51-60, 2014.

33. Coker-Gurkan A, Arisan ED, Obakan P, Guvenir E and Unsal NP: Inhibition of autophagy by 3-MA potentiates purvalanol-induced apoptosis in Bax deficient HCT 116 colon cancer cells. Exp Cell Res 328: 87-98, 2014.

34. Zhai H, Song B, Xu X, Zhu W and Ju J: Inhibition of autophagy and tumor growth in colon cancer by miR-502. Oncogene 32: 1570-1579, 2013

35. Zou Z, Wu L, Ding H, Wang Y, Zhang Y, Chen X, Chen X, Zhang CY, Zhang Q and Zen K: MicroRNA-30a sensitizes tumor cells to cis-platinum via suppressing beclin 1-mediated autophagy. J Biol Chem 287: 4148-4156, 2012.

36. Chatterjee A, Chattopadhyay D and Chakrabarti G: miR-17-5p downregulation contributes to paclitaxel resistance of lung cancer cells through altering beclin1 expression. PLoS One 9: e95716, 2014

37. Chen Y, Li X, Wu X, He C, Guo L, Zhang S, Xiao Y, Guo W and Tan B: Autophagy-related proteins LC3 and Beclin-1 impact the efficacy of chemoradiation on esophageal squamous cell carcinoma. Pathol Res Pract 209: 562-567, 2013

38. Fujiwara K, Iwado E, Mills GB, Sawaya R, Kondo S and Kondo Y: Akt inhibitor shows anticancer and radiosensitizing effects in malignant glioma cells by inducing autophagy. Int $\mathbf{J}$ Oncol 31: 753-760, 2007.

39. Kim EJ, Jeong JH, Bae S, Kang S, Kim CH and Lim YB: mTOR inhibitors radiosensitize PTEN-deficient non-small-cell lung cancer cells harboring an EGFR activating mutation by inducing autophagy. J Cell Biochem 114: 1248-1256, 2013. 